\title{
Characteristics of Clostridium difficile isolates and the burden of hospital- acquired Clostridium difficile infection in a tertiary teaching hospital in Chongqing, Southwest China
}

\author{
Wei Dai ${ }^{1+}$, Tianxiang Yang ${ }^{2+}$, Li Yan', Siqiang Niu' ${ }^{1}$, Chuanming Zhang ${ }^{1}$, Jide Sun ${ }^{1}$, Zhu Wang ${ }^{1}$ and Yun Xia ${ }^{{ }^{*}}$
}

\begin{abstract}
Background: Clostridium difficile infection (CDI), especially hospital-acquired Clostridium difficile infection (HA-CDI), continues to be a public health problem and has aroused great concern worldwide for years. This study aimed to elucidate the clinical and epidemiological features of HA-CDI and the characteristics of C.difficile isolates in Chongqing, Southwest China.

Methods: A case-control study was performed to identify the clinical incidence and risk factors of HA-CDI. C. difficile isolates were characterised by polymerase chain reaction (PCR) ribotyping, multilocus sequence typing (MLST), toxin gene detection and antimicrobial susceptibility testing.

Results: Of the 175 suspicious patients, a total of 122 patients with antibiotic-associated diarrhea (AAD) were included in the study; among them, 38 had HA-CDI. The incidence of AAD and HA-CDI was 0.58 and 0.18 per 1000 patient admissions, respectively. Chronic renal disease and cephalosporin use were independent risk factors for HACDI. Fifty-five strains were assigned into 16 sequence types (STs) and 15 ribotypes (RTs). ST2/RT449 (8, 14.5\%) was the predominant genotype. Of the 38 toxigenic isolates, $A+B+C D T$ - isolates accounted for most $(34,89.5 \%)$ and 1 $\mathrm{A}+\mathrm{B}+\mathrm{CDT}+$ isolate emerged. No isolate was resistant to vancomycin, metronidazole or tigecycline, with A-B-CDTbeing more resistant than $\mathrm{A}+\mathrm{B}+\mathrm{CDT}$ -

Conclusions: Different genotypes of $C$. difficile strains were witnessed in Chongqing, which hinted at the necessary surveillance of HA-CDI. Adequate awareness of patients at high risk of HA-CDI acquisition is advocated and cautious adoption of cephalosporins should be highlighted.
\end{abstract}

Keywords: Hospital-acquired Clostridium difficile infection, Risk factor, Genotyping, Antimicrobial resistance

\footnotetext{
*Correspondence: 75538345@qq.com

'Wei Dai and Tianxiang Yang contributed equally to this work.

'Department of Laboratory Medicine, The First Affiliated Hospital of

Chongqing Medical University, No.1 Youyi Road, Yuzhong District,

Chongqing 400016, People's Republic of China

Full list of author information is available at the end of the article
}

(c) The Author(s). 2020 Open Access This article is licensed under a Creative Commons Attribution 4.0 International License, which permits use, sharing, adaptation, distribution and reproduction in any medium or format, as long as you give appropriate credit to the original author(s) and the source, provide a link to the Creative Commons licence, and indicate if changes were made. The images or other third party material in this article are included in the article's Creative Commons licence, unless indicated otherwise in a credit line to the material. If material is not included in the article's Creative Commons licence and your intended use is not permitted by statutory regulation or exceeds the permitted use, you will need to obtain permission directly from the copyright holder. To view a copy of this licence, visit http://creativecommons.org/licenses/by/4.0/ The Creative Commons Public Domain Dedication waiver (http://creativecommons.org/publicdomain/zero/1.0/) applies to the data made available in this article, unless otherwise stated in a credit line to the data. 


\section{Background}

As a successful nosocomial pathogen, toxin-producing C. difficile has caused approximately $10-30 \%$ healthcareassociated infections [1, 2]. Increased incidence and severity of Clostridium difficile infection (CDI) have been witnessed in Europe and North America in recent decades $[3,4]$. However, in developing countries, due to the poor awareness of healthcare workers and limited capacity of laboratory diagnosis, the potential public threat of CDI has not been fully recognized. A recent random-effects study including 37,663 patients reported a similar incidence rate of CDI in Asia in comparison with North America and Europe. Significant regional variation has been revealed and when compared with the Middle East and South Asia, East Asia was exposed to the highest CDI prevalence of $19.5 \%$ [5], which necessitated good awareness and surveillance of CDI in this area.

However, unlike the rest of East Asia, limited data have focused on the burden of CDI in China. Although few regional studies alarmed that the hyper-virulent $C$. difficile strain ST-1 (BI/NAP1/027), an epidemic strain in Europe and North America, has emerged in Chinese hospital settings, recent reports revealed that ST35, ST37 and ST3 were the most prevalent genotypes in mainland China [6, 7]. Moreover, in consideration of the complex personnel mobility in medical institutions, the majority of CDI is hospital-acquired, and nosocomial transmission of $C$. difficile contributes greatly to the spread of different genotypes. Recently, whole genome sequencing (WGS) identified the dissemination and spread of $C$. difficile ribotype 027 (RT027) and sequence type 081 (ST081) in two Chinese hospitals [8, 9]. Therefore, a better understanding of regional epidemiology is helpful to guide priorities for the management of hospital-acquired Clostridium difficile infection (HACDI). Although many studies have explored the CDI situation in China, the lack of epidemiological data in blind areas impedes a full understanding of CDI in this country. To the best of our knowledge, this is the first study of HA-CDI in Chongqing, a provincial administrative unit in Southwest China [6]. Our study was initiated to investigate the impact of HA-CDI by identifying its prevalence, determine the risk factors for the acquisition of this dilemma in patients with antibiotic-associated diarrhea (AAD), reveal the mortality of HA-CDI in this teaching hospital and inquire into the molecular epidemiology and antimicrobial resistance of C.difficile isolates found in this study.

\section{Methods}

\section{Study design}

A case-control study was conducted from June 2014 to March 2016 in the First Affiliated Hospital of Chongqing
Medical University, a tertiary teaching hospital with 3200 beds, which is the surveillance center of antimicrobial resistance in Chongqing. Unformed stools of inpatients suffering from diarrhea were collected for toxigenic culture of $C$. difficile. After medical chart screening, patients who were hospitalized for more than or equal to 7 days and who were administered antibiotics before diarrhea were included in this study. According to the results from toxigenic culture, patients diagnosed with HA-CDI were enrolled in the case group, while patients diagnosed with non- $C$. difficile AAD were enrolled in the control group (Fig. 1). Clinical data including demography, chronic underlying disease, comorbidities, medication prior to the onset of diarrhea, in-hospital recurrence and mortality were retrieved by electronic medical charts.

\section{Definitions}

AAD was diagnosed when a hospitalized patient suffered from unexplained diarrhea in association with the administration of antibiotics during current hospitalization [1].

CDI was diagnosed when a patient with diarrhea was positive for a toxin-producing $C$. difficile strain in stool culture.

HA-CDI was the case with CDI confirmed at least 48 $\mathrm{h}$ after admission.

Four severity levels of CDI (mild, moderate, severe and complicated) introduced by Leffler et al. [10] were adopted to judge the clinical manifestations of patients in this study. Clinical outcomes of HA-CDI cases were classified into three categories: a. symptomatic recovery, b. CDI symptomatic recurrence while in hospital, c. inhospital death after the diagnosis of HA-CDI.

\section{Microbiological testing}

Unformed stool samples treated with alcohol shock were inoculated onto cycloserin-cefoxitin-fructose agar (CCFA) and cultured anaerobically in $37^{\circ} \mathrm{C}$ for 5 days. The colonies suspected as $C$. difficile were subjected to mass spectrometry (Vitek MS, bioMerieux, France). C. difficile strains were assayed for toxin $\mathrm{A}$ and toxin $\mathrm{B}$ antigen by enzyme linked fluorescent assay (ELFA) (Vidas mini, bioMerieux, France) performed on culture supernatants in vitro. DNA of the $C$. difficile strain was extracted and polymerase chain reaction (PCR) was performed to detect the presence of toxin genes ( $t c d A$, $t c d B)$, the regulatory gene $(t c d C)$ and binary toxin genes $(c d t A$ and $c d t B)$ as previously reported $[11,12]$. The flow diagram of the laboratory diagnosis of $C$. difficile infection by toxigenic culture was summarized in Fig. 1. Antimicrobial susceptibilities of $C$. difficile to seven antibiotics (vancomycin, metronidazole, rifampin, levofloxacin, erythromycin, clindamycin and tigecycline) 


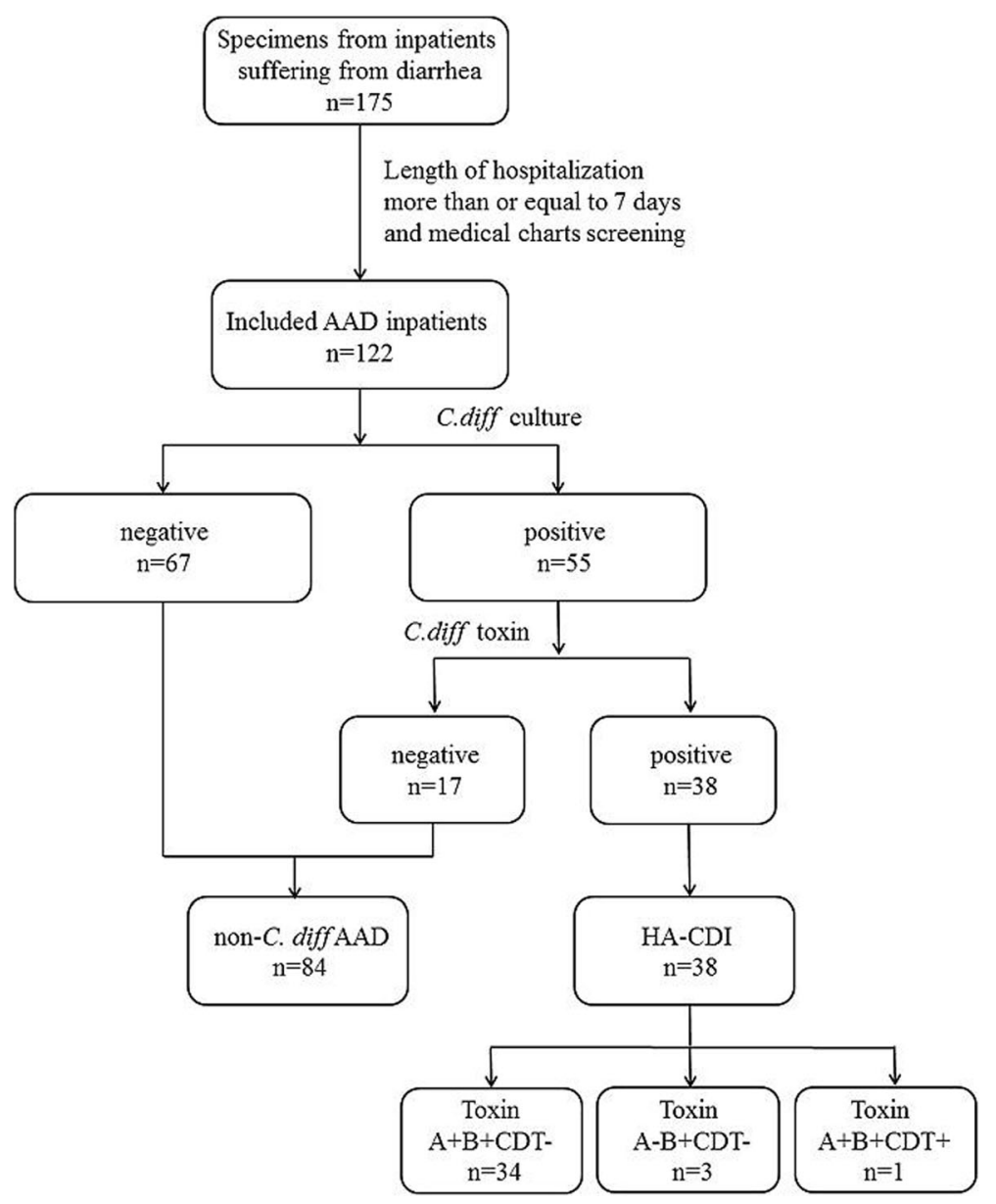

Fig. 1 The flow diagram of data collection and laboratory diagnosis of $C$. difficile infection. Stool samples from patients with antibiotic-associated diarrhea (AAD) were cultured for $C$. difficile. Isolates from culture positive patients were assayed for toxin $A / B$ in vitro and if positive for toxin in vitro, cases were identified as HA-CDI. Cases were further categorized by PCR amplification of isolates to determine their toxin gene profiles (toxin A, B, CDT). This surveillance was carried out from June 2014 to March 2016 in the First Affiliated Hospital of Chongqing Medical University

were tested by the agar dilution method as recommended by the Clinical and Laboratory Standards Institute (CLSI) M11-A8 [13]. Breakpoints for clindamycin and metronidazole were according to CLSI recommendations for anaerobic bacteria [14], while those for rifampin, erythromycin and levofloxacin were adopted on the basis of the suggestion made by Lidan $\mathrm{C}$ et al. [15]. Interpretation criteria of vancomycin and tigecycline were according to European Committee on Antimicrobial Susceptibility Testing (EUCAST) recommendations [16].

\section{Molecular typing analysis}

Multilocus sequence typing (MLST) was performed by sequencing seven house-keeping genes of $C$. difficile ( $a d k, \operatorname{atp} A, d x r, \operatorname{gly} A, \operatorname{rec} A, \operatorname{sod} A$ and $t p i)$ as previously reported by Griffiths D et al. [11]. Sequence types (STs) and clades of $C$. difficile strains were confirmed by querying on http://pubmlst.org/ website. A minimum spanning tree generated from BioNumerics version 7.6 was used to show the genetic diversity of the MLST data derived from this study.

Capillary gel electrophoresis-based PCR ribotyping was implemented according to a previous report by Fawley WN et al. [17]. Ribotypes were identified by querying on the WEBRIBO web-based database (http://webribo. ages.at). The novel ribotype was named as "Chongqing Ribotype" (CQR) plus two Arabic numbers (e.g., CQR01).

\section{Statistical analysis}

Patients with a length of hospitalization more than or equal to 7 days were included in the statistical analysis. A univariate analysis was initially conducted to determine the potential risk factors for the acquisition of HACDI by comparing the HA-CDI group with the non- $C$. difficile AAD group. Categorical variables were compared by use of chi-square or Fisher's exact test. Odds 
ratios (ORs) and 95\% confidence intervals (CIs) were calculated to evaluate the strength of any association. Variables with a $P$ value of $<0.10$ in the univariate test were included in a multivariate one-step logistic remodel. A two-tailed $P$-value of $<0.05$ was considered to be statistically significant. All of the statistical calculations were performed with standard programs in SPSS v.21.0 (SPSS, Chicago, IL, USA) [18].

\section{Results}

\section{The prevalence of AAD and HA-CDI}

During the surveillance period, a total of 211,536 patients were hospitalised and 91,800 received antibiotic treatment. A total of 175 patients developed diarrhea but only $122(69.7 \%)$ received antibiotic treatment before that thus were included in this study as AAD patients. AAD developed in $1.3 \%$ of antibiotic-treated inpatients and had an incidence of 0.58 per 1000 patient admissions in this hospital. Among the 122 AAD patients, $C$. difficile was isolated from the specimens of 55 respondents, and 38 (31.1\%) were positive for toxigenic C. difficile culture and diagnosed with HA-CDI, yielding an incidence of $0.41 \mathrm{HA}-\mathrm{CDI}$ per 1000 antibiotic-treated patients and 0.18 HA-CDI per 1000 patient admissions. Among the HA-CDI patients, the average age was $54.5 \pm 17.4$ years and $60.5 \%$ of the patients were older than 65 years. Twenty-eight $(73.7 \%)$ were males. A majority of HA-CDI patients were from surgical wards. The median time between admission and the onset of diarrhea was 7 days, while that between admission and diagnosis of HA-CDI was 14 days. The severity of patients with HA-CDI ranged from mild to moderate, no severe HA-CDI case was recorded in this work. Thirty-five out of 38 (92.1\%) HA-CDI patients had symptomatic recovery and no recurrence was noted. Three $(7.9 \%)$ patients died during hospitalisation but not because of HA-CDI.

\section{Clinical characteristics and risk factors for HA-CDI}

The clinical characteristics and risk factors for HA-CDI in AAD patients were summarized in Table 1. No significant demographic differences (such as age and gender) were observed between the two groups. Compared with the non- $C$. difficile AAD cases, HA-CDI patients were significantly more likely to have surgery in the last 6 months and more prone to suffering from chronic renal disease, pulmonary infection and hypoalbuminemia on admission. Exposure to chemotherapy, cephalosporins, metronidazole and proton pump inhibitors (PPIs) use were more frequent in patients with HA-CDI. Multivariate analysis showed that chronic kidney diseases (OR, 4.275; 95\% CI, 1.154-15.839; $P=.030$ ) and cephalosporins exposure (OR, 8.840; 95\% CI, 2.807-27.836; $P=.000)$ were independent risk factors for HA-CDI acquisition in AAD patients.

\section{Genotyping characteristics of $C$. difficile isolates}

In total, 55 non-duplicated strains were isolated and assigned to 16 genotypes by MLST. ST2 $(n=9,16.4 \%)$ was the most common genotype, followed by ST39 $(n=$ $7,12.7 \%)$ and ST37 ( $n=6,10.9 \%)$. ST35, ST54 and ST205 were commonly detected. A novel genotype ST352 was found. A majority $(n=40,72.7 \%)$ of isolates were categorized as clade 1 , followed by clade $4(n=13$, $23.6 \%)$ and clade $3(n=2,3.6 \%)$. The minimum spanning tree showed the relationship of ST types in Fig. 2.

All 55 isolates recovered were finally assigned to 15 PCR ribotypes (RTs) with 8 known RTs and 7 novel RTs. RT449 $(n=10,18.2 \%)$, RT085 $(n=7,12.7 \%)$, RT012 $(n=6,10.9 \%)$, and RT017 $(n=5,9.1 \%)$ were the main RTs. Of the seven novel ribotypes, CQR03 and CQR04 exhibited a high prevalence $(n=5,9.1 \%)$. None of the isolates belonged to RT027 or RT078. Superimposition of the percentage diagram with the time of $C$. difficile detection by season revealed predominant proportions of RT449, RT085, and RT012 in this study (Fig. 3). Looking through the data derived from the two methods, ST2/RT449 (8, 14.5\%) was the predominant genotype, followed by ST39/ RT085 (7, 12.7\%), ST54/RT012 (5, 9\%) and ST37/ RT017 (5, 9\%). Exclusive correlations were found among three groups: ST26/RT39/2, ST39/RT085 and ST3/RT456.

\section{Toxigenic characteristics and their correlation with the genotypes of isolates}

In all 55 isolates, an exclusive correlation was found between toxin types and genotypes (seen in the Additional file 1). All the ST39/RT085 isolates were A-B-, while all the RT012 isolates were toxin-producing (A+ $\mathrm{B}+)$. Of the 55 isolates, 38 (69.1\%) were toxin-producing, including 34 (89.5\%) with toxigenic type A + B + CDT-, 3 (7.9\%) with toxigenic type A-B + CDT- and 1 (2.6\%) with toxigenic type $\mathrm{A}+\mathrm{B}+\mathrm{CDT}+$.

Of the 38 toxigenic isolates, ST2/RT449 $(8,21.1 \%)$ and ST54/RT012 $(5,13.2 \%)$ were the predominant toxigenic genotypes. In MLST, ST2 $(n=9,23.7 \%)$ was most frequently detected, followed by ST37 $(n=6$, $15.8 \%)$, ST54 $(n=5,13.2 \%)$ and ST35 $(n=5,13.2 \%)$. PCR ribotyping found 11 ribotypes, including 6 known ribotypes and 5 novel ribotypes. RT449 $(n=9$, $23.7 \%)$ RT012 $(n=6,15.8 \%)$ and RT017 $(n=5$, $13.2 \%)$ were the most frequent ribotypes. The CDT+ strain was assigned to the genotype of ST5/RT498 in clade 3 (Table 2).

Antimicrobial susceptibility between genotypes and toxin types of isolates

The minimum inhibitory concentrations (MICs) of seven antimicrobial agents for 55 non-duplicated strains were 
Table 1 Statistical analysis for risk factors of hospital-acquired C. difficile infection (HA-CDI) in AAD patients

\begin{tabular}{|c|c|c|c|c|c|c|}
\hline \multirow[t]{2}{*}{ Variable } & \multirow{2}{*}{$\begin{array}{l}\text { HA-CDI } \\
(n=38) \\
\text { No.(\%) }\end{array}$} & \multirow{2}{*}{$\begin{array}{l}\text { Non-C. } \\
\text { difficile } \\
\text { AAD } \\
(n=84) \\
\text { No.(\%) }\end{array}$} & \multicolumn{2}{|c|}{ Univariate analysis } & \multicolumn{2}{|c|}{ Multivariate analysis } \\
\hline & & & $P$ value & OR & $P$ value & $\mathrm{OR}(95 \% \mathrm{Cl})$ \\
\hline \multicolumn{7}{|l|}{ Demographic data } \\
\hline Male gender & $28(73.7)$ & $62(73.8)$ & 0.988 & 1.006 & & \\
\hline Elderly( $\geq 65$ years) & $23(60.5)$ & $37(44.0)$ & 0.795 & 0.949 & & \\
\hline Admission to ICU & $16(42.1)$ & $38(45.2)$ & 0.747 & 0.931 & & \\
\hline \multicolumn{7}{|l|}{ Comorbidities } \\
\hline Chronic kidney diseases & $12(31.6)$ & $12(14.3)$ & $0.026^{*}$ & 2.211 & $0.030^{*}$ & $4.275(1.154-15.839)$ \\
\hline Coronary heart disease & $3(7.9)$ & $5(6.0)$ & 0.703 & 1.326 & & \\
\hline Diabetes mellitus & $8(21.1)$ & 16(19.0) & 0.796 & 1.105 & & \\
\hline Hypertension & 13(34.2) & $30(35.7)$ & 0.872 & 0.958 & & \\
\hline Hepatic disease & $12(31.6)$ & 15(17.9) & 0.091 & 1.768 & & \\
\hline Malignancy & 7(18.4) & 15(17.9) & 0.940 & 1.032 & & \\
\hline Surgery in the past 6 months & $16(42.1)$ & 16(19.0) & $0.007^{*}$ & 2.211 & & \\
\hline \multicolumn{7}{|l|}{ Diagnosis on admission } \\
\hline Hypoalbuminaemia & 25(65.8.) & 16(19.0) & $0.000^{*}$ & 3.454 & & \\
\hline Urinary tract infection & $5(13.2)$ & $8(9.5)$ & 0.541 & 1.382 & & \\
\hline Pulmonary infection & $22(57.9)$ & 25(29.8) & $0.003^{*}$ & 1.945 & & \\
\hline Bloodstream infection & $2(5.3)$ & $7(8.3)$ & 0.719 & 0.632 & & \\
\hline \multicolumn{7}{|c|}{ Medication prior to the onset of diarrhea during hospitalization } \\
\hline Glucocorticoids & 12(31.6) & $21(25.0)$ & 0.449 & 1.263 & & \\
\hline Chemotherapy & 7(18.4) & $3(3.6)$ & $0.010^{*}$ & 5.158 & & \\
\hline PPIs & $20(52.6)$ & $64(76.2)$ & $0.009^{*}$ & 0.691 & & \\
\hline Penicillin & $17(44.7)$ & $41(48.8)$ & 0.677 & 0.917 & & \\
\hline Cephalosporins & $23(60.5)$ & 10(11.9) & $0.000^{*}$ & 5.084 & $0.000^{*}$ & $8.840(2.807-27.836)$ \\
\hline Carbapenems & $16(42.1)$ & $39(46.4)$ & 0.677 & 0.907 & & \\
\hline Aminoglycosides & $5(13.2)$ & $7(8.3)$ & 0.513 & 1.579 & & \\
\hline Fluoroquinolones & $5(13.2)$ & 15(17.9) & 0.605 & 0.737 & & \\
\hline Glycopeptides & $15(39.5)$ & $30(35.7)$ & 0.690 & 1.105 & & \\
\hline Metronidazole & 13(34.2) & $9(10.7)$ & $0.020^{*}$ & 3.193 & & \\
\hline
\end{tabular}

HA-CDI hospital-acquired C. difficile infection, $A A D$ antibiotic-associated diarrhea, $O R$ odds ratio, $C l$ confidence interval, $P P I s$ Proton pump inhibitors, ${ }^{*} p<0.05$

summarized in Table 3 (raw data shown in Additional file 2). Eight (14.5\%) isolates were found to be multidrug resistant (MDR). None of the isolates were resistant to vancomycin, metronidazole or tigecycline, while high resistance to erythromycin and clindamycin was observed with rates of 87.3 and $61.8 \%$, respectively. Twelve point seven percent of the isolates were resistant to rifampin and $14.5 \%$ were resistant to levofloxacin.

In comparison with $\mathrm{A}+\mathrm{B}+$ isolates and $\mathrm{A}-\mathrm{B}+$ isolates, higher resistance rates of $\mathrm{A}-\mathrm{B}$ - isolates to rifampin, levofloxacin, erythromycin and clindamycin were revealed. Relatively low resistance rates of $\mathrm{A}+\mathrm{B}+$ isolates to rifampin $(2.9 \%)$ and levofloxacin (2.9\%) were observed.
Varied antimicrobial phenotypes demonstrated in different RTs. The resistance rates of RT012 to erythromycin and clindamycin (100 and 83.3\%, respectively) were higher than those of RT449. All of the RT017 isolates were co-resistant to erythromycin and clindamycin. Sixty percent of the RT017 isolates were co-resistant to erythromycin, clindamycin and levofloxacin.

\section{Discussion}

Enhanced molecular diagnostic and antibiotic treatment strategies promote the continuous evolution of the knowledge of CDI epidemiology. Geographical heterogeneity and transcontinental dissemination have aroused 


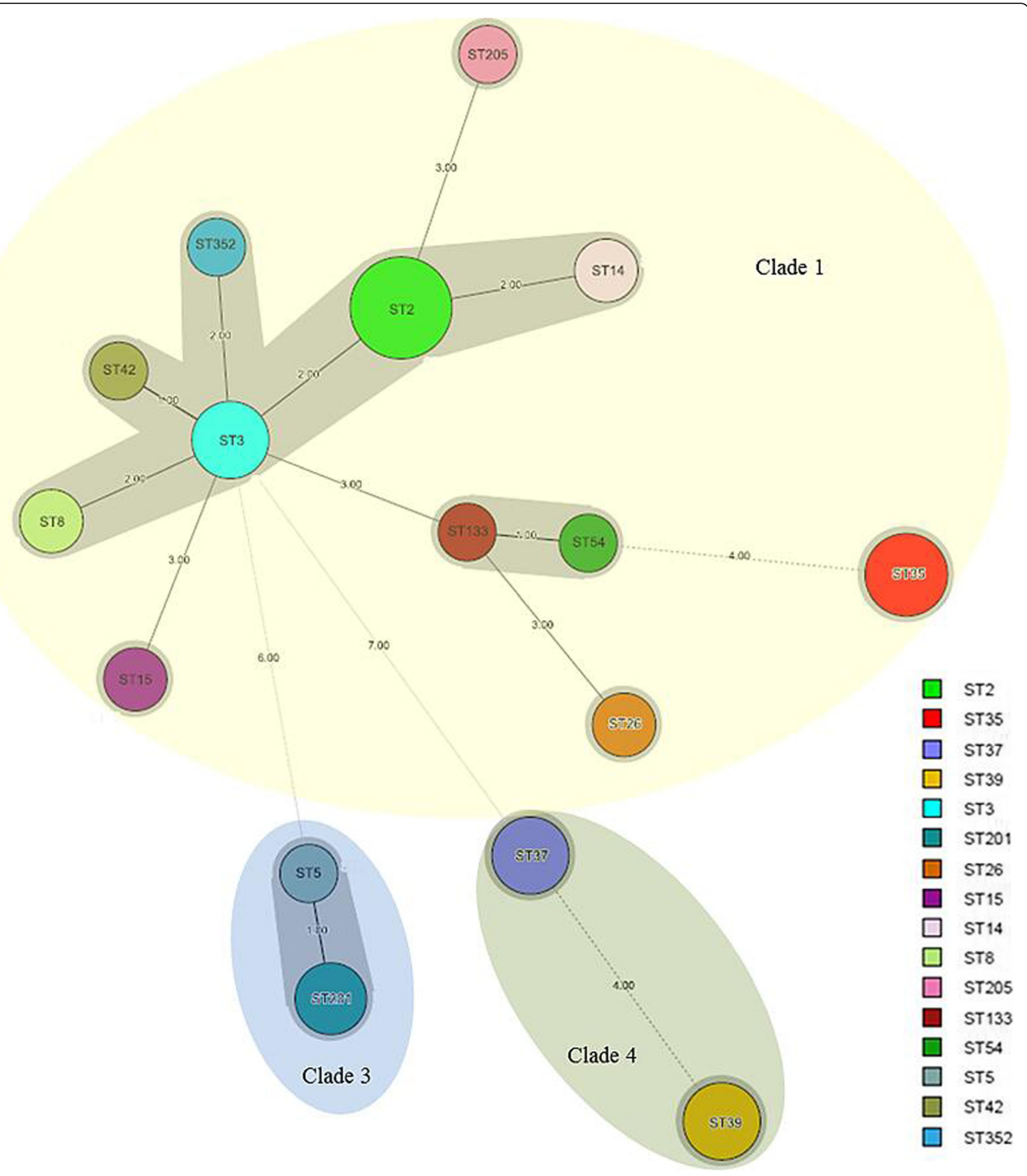

Fig. 2 The minimum spanning tree for displaying the distribution and relationship of MLST sequence types found in this study. The circle size represented the number of isolates of each corresponding type. The figure on the line linking two circles demonstrated the number of different loci between them. The types with less than or equal to two different loci were covered by the gray area. Three clades were indicated by colourful areas

more concerns about regional CDI surveillance. In China, although the state of dilemma introduced by CDI has been documented before $[6,7]$, data are lacking in the central and western regions. To fill gaps in the epidemiological territory of CDI in China, the results in this study presented basic knowledge of the prevalence and mortality of HA-CDI, and helped to improve the recognition of patients at high risk for HA-CDI 


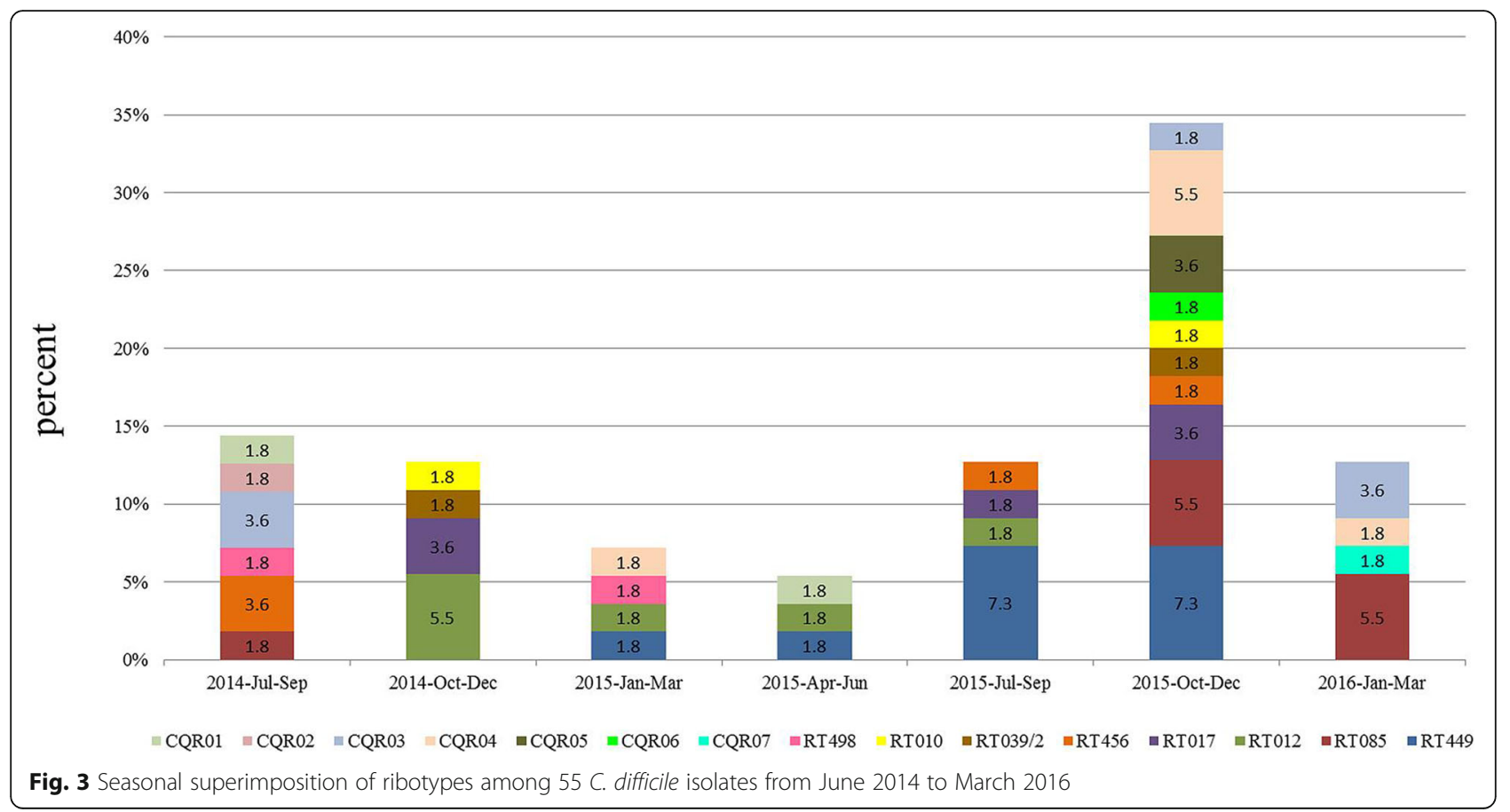

Table 2 Typing results and toxin genotypes of $55 \mathrm{C}$. difficile isolates

\begin{tabular}{|c|c|c|c|c|c|c|c|c|}
\hline Clades & MLST & CGE & $t c d A$ & $t c d B$ & $\operatorname{tcdC}$ & $c d t A$ & $C d t B$ & No.of isolates \\
\hline \multirow[t]{15}{*}{1} & \multirow[t]{2}{*}{ ST8 } & CQRO2 & + & + & + & - & - & 1 \\
\hline & & RT010 & + & + & + & - & - & 1 \\
\hline & ST3 & RT456 & + & + & + & - & - & 4 \\
\hline & ST42 & CQR01 & + & + & + & - & - & 2 \\
\hline & ST54 & RT012 & + & + & + & - & - & 5 \\
\hline & \multirow[t]{2}{*}{ ST2 } & RT449 & + & + & + & - & - & 8 \\
\hline & & CQR07 & + & + & + & - & - & 1 \\
\hline & ST35 & CQRO4 & + & + & + & - & - & 5 \\
\hline & ST133 & RT449 & + & + & + & - & - & 1 \\
\hline & ST14 & CQR05 & + & + & + & - & - & 2 \\
\hline & ST205 & CQRO3 & - & - & - & - & - & 5 \\
\hline & ST26 & RT39/2 & - & - & - & - & - & 2 \\
\hline & \multirow[t]{2}{*}{ ST15 } & RT449 & - & - & - & - & - & 1 \\
\hline & & RT010 & - & - & - & - & - & 1 \\
\hline & ST352 & CQR06 & - & - & - & - & - & 1 \\
\hline \multirow[t]{2}{*}{3} & ST5 & RT498 & + & + & + & + & + & 1 \\
\hline & ST201 & RT498 & + & + & + & - & - & 1 \\
\hline \multirow[t]{4}{*}{4} & \multirow[t]{3}{*}{ ST37 } & RT017 & - & + & + & - & - & 3 \\
\hline & & RT017 & + & + & + & - & - & 2 \\
\hline & & RT012 & + & + & + & - & - & 1 \\
\hline & ST39 & RT085 & - & - & - & - & - & 7 \\
\hline
\end{tabular}

MLST multilocus sequence typing, CGE capillary gel electrophoresis, CQR Chongqing Ribotype New-ribo-type found in Chongqing, ST sequence type, $R T$ ribotype acquisition and to guide antibiotic stewardship initiatives of HA-CDI in this tertiary teaching hospital in Southwest China.

A survey focusing on antibiotic consumption in specialized public hospitals in 30 provinces in mainland China showed a decrease in the percentage of antibiotic use in inpatients in Chongqing, from 78.84 to 54.93\% [19]. The present study found the ratio of antibiotic use in inpatients was $43.4 \%$. Despite being relatively low and comparable to the previous data reported by Zhou et al. [20], this percentage, to a large extent, surpassed the recommendation of $30 \%$ by World Health Organization (WHO). Previous studies have reported varied frequencies of AAD from 0.57 to $14.9 \%$ in different populations $[21,22]$. The correlation between antibiotic use and the prevalence of $\mathrm{AAD}$ in Chongqing was previously unknown. The present study witnessed a moderate prevalence of $\mathrm{AAD}$ in $0.13 \%$ (a much lower rate) of antibiotictreated inpatients. One possible explanation is that a majority of the patients in this cohort were from surgical wards and received antibiotics simply for perioperative prophylaxis.

In this investigation, HA-CDI accounted for $31.1 \%$ of AAD, which was consistent with previous reports [20, 23]. The high prevalence of CDI among AAD is always a major concern worldwide [24]. To prevent CDI from AAD, external interventions and internal defense mechanisms should work cooperatively. Our previous study has shown that interleukin-27 (IL-27)/IL-27 receptor signaling provides protection against $C$. difficile-induced colitis in AAD patients [25]. A recent systematic review 


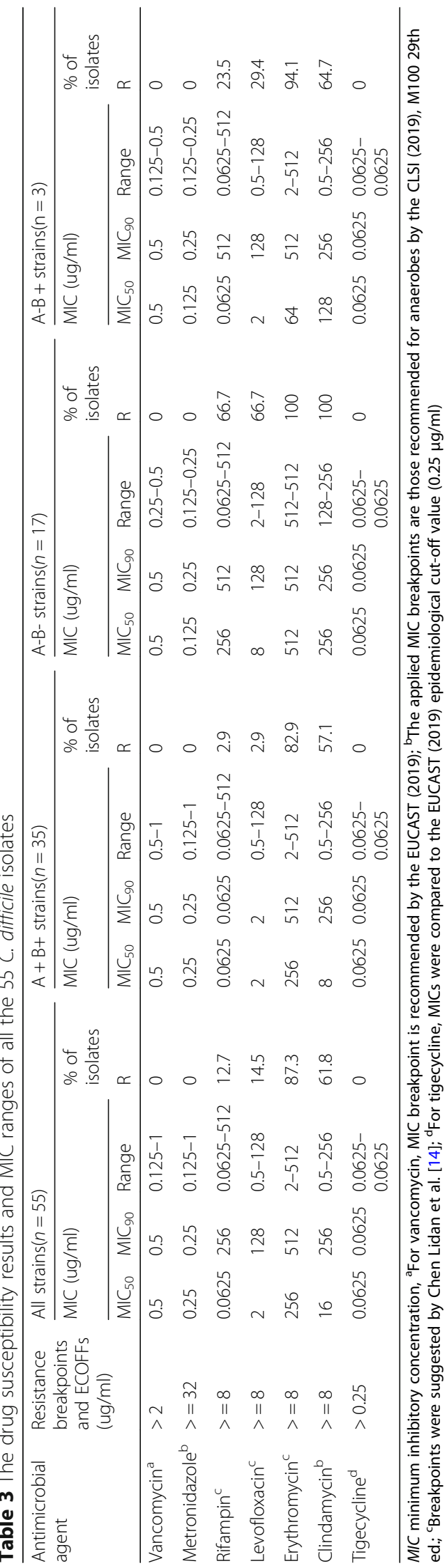


and meta-analysis reported an incidence of 0.32 cases of CDI per 1000 patient admissions in Asia [5] and a similar result was verified in a 7-year retrospective study in a large university hospital in Eastern China [26]. This study reported a relatively low incidence of 0.18 per 1000 patient admissions, probably due to inadequate awareness of CDI among clinicians, low sensitivity of stool anaerobic culture for $C$. difficile detection, and low testing frequency [27]. Another possible reason is the missing information of a proportion of inpatients who might develop CDI after discharge.

As is well known, the use of antibiotics may cause CDI [28], but the case-control design focusing on the difference between antibiotic group and non-antibiotic group may have the trends to overestimate the impact of antibiotic exposure on the acquisition of HA-CDI. To explore the specific reasons leading to $\mathrm{CDI}$, this study set up a comparison between the HA-CDI group and the non-C. difficile AAD group in AAD patients to identify which antibiotics or predictors were associated with a high risk for HA-CDI. Although many risk factors were revealed in univariate analysis, only two independent risk factors, cephalosporin use prior to the onset of diarrhea and chronic kidney diseases were identified for patients with HA-CDI when compared to non-C.difficile AAD, which is consistent with previous reports [29-31] and not difficult to explain. In addition to the cephalosporins known to all [32], chronic renal disease may cause poor excretion of antibiotic agents, high concentration in blood and finally, the imbalance of bacterial flora in the gut.

Age over 65 years was not associated with an increased risk for HA-CDI. Suffering from HA-CDI in younger age was observed in this study. Similar results have been reported in several previous studies in mainland China and France [23, 29, 33]. Therefore, it is necessary to consider the age threshold in the recognition of inpatients at high risk for HA-CDI in different settings. Massive consumption of antibiotics by university students has been reported nationwide in China [34]. Accumulative effects of antibiotic consumption may contribute partly to the acquisition of CDI in younger age, which deserves more attention. Moreover, ageing is accompanied by changes in the gut microbiome [35], and it is speculated that it is not the age threshold, but the gut microbial structure that truly participates in the priming of HACDI.

To reveal the epidemiology of CDI in mainland China, in this non-outbreak situation, specific genotypes of toxigenic C. difficile strains were observed. ST2 was the most predominant genotype, while recent studies reported that ST54, ST3 and ST37 were the most prevalent genotypes in mainland China [6, 33, 36-38]. Noteworthily, in addition to ST54 and ST3, ST35 also emerged both in this work and another inspection in Yunnan [39], a province bordering Chongqing, witnessing the spread of this toxin genotype over provinces in China. Toxigenic RT449 with a high prevalence in this work was not reported previously, and its predominant proportion may indicate an upcoming outbreak. One $C$. difficile isolate was positive for binary toxin and belonged to ST5/RT498 in clade 3. Although the CDT+ strain appeared less frequently in Asia, this was not the first report of this toxin genotype in China. ST5 accounted for $83.7 \%$ of binary toxin gene-positive strains in a survey conducted by Chen et al. [40] in 2018. Data on $C$. difficile strains in clade 3 with binary toxins are not well documented in mainland China. WGS of three clade $3 \mathrm{C}$. difficile strains carrying binary toxin genes in a university hospital found that clade 3 has unusual clade-specific PaLoc characteristic of Tn6218 insertion, which may be the main feature to distinguish clade 3 from other $C$. difficile [41]. The identification of seven novel RTs indicated the diversity of $C$. difficile strains in this hospital.

Despite the fact that clinical C. difficile strains with hetero-resistance or high-level resistance to metronidazole were reported in China [20,33], the present study failed to identify strains resistant to metronidazole, vancomycin or tigecycline, indicating that these three antibiotic agents still seem to be appropriate for empirical treatment of HA-CDI. In addition, toxin types were associated with antibiotic resistance phenotypes. A-Bstrains were more resistant than $\mathrm{A}+\mathrm{B}+$ strains, while the latest data from two hospitals in Shangdong illustrated that non-toxigenic strains were more sensitive [42].

Our study has some limitations. First, these results were derived from a single-center. Widely recommended detection schemes, two-step and three-step methods for the diagnosis of CDI, were implemented in many laboratories in China, but anaerobic culture was not the choice for the final confirmation. This may be one of the causes for the lack of epidemiological data in this country. To obtain surveillance data for CDI, a network of reference or central laboratories such as that found in Europe is needed [43]. Second, this study failed to track clinical treatments of HA-CDI, but most patients recovered from diarrhea after the discontinuation of antibiotic therapy. Third, highly sensitive tests, such as the nucleic acid amplification test (NAAT) or the glutamate dehydrogenase $(\mathrm{GDH})$ screening test, were not performed in this study.

\section{Conclusions}

In summary, this study presented a comprehensive survey of HA-CDI and AAD in Chongqing, Southwest China. The burdens of HA-CDI and AAD were 
moderate. Inpatients undergoing cephalosporins therapy and suffering from chronic kidney diseases, who are thus at high risk for HA-CDI, deserve more attention. The regional diversity of $C$. difficile strains in genotype necessitates good awareness of HA-CDI by holding an evolving insight into the surveillance of this adverse event. In addition to the notorious genotypes, sufficient attention should be paid to the relatively rare toxigenic strains found in this report, such as ST5/RT498, during molecular epidemiology monitoring.

\section{Supplementary information}

Supplementary information accompanies this paper at https://doi.org/10. 1186/s12879-020-05014-6.

Additional file 1 The detection results and department sources of $55 \mathrm{C}$. difficile strains.

Additional file 2. The MICs of seven antimicrobial agents for isolates included in this study.

\section{Abbreviations}

CDI: Clostridium difficile infection; HA-CDI: Hospital-acquired Clostridium difficile infection; AAD: Antibiotic-associated diarrhea; PCR: Polymerase Chain Reaction; RT: Ribotype; MLST: Multilocus sequence typing; CCFA: Cycloserincefoxitin-fructose agar; ELFA: Enzyme linked fluorescent assay; CLSI: Clinical and Laboratory Standards Institute; EUCAST: European Committee on Antimicrobial Susceptibility Testing; ST: Sequence type; CQR: Chongqing Ribotype; OR: Odds ratio; Cl: Confidence interval; PPIs: Proton pump inhibitors; MIC: Minimum inhibitory concentration; MDR: Multidrug resistant; WHO: World health organization; IL: Interleukin; CGE: Capillary gel electrophoresis; WGS: Whole genome sequencing; NAAT: Nucleic acid amplification test; GDH: Glutamate dehydrogenase

\section{Acknowledgements}

Not applicable.

\section{Authors' contributions}

DW performed the molecular typing analysis and was a major contributor in writing the manuscript. YTX referred to the electronic medical records and accomplished the detection of toxin genes. NSQ completed statistical analysis. YL, ZCM, SJD and WZ carried out the routine sample tests including anaerobic culture and toxin detection, collected isolates and implemented the antibiotic susceptibility test. XY finished the study design. All authors read and approved the final manuscript.

\section{Funding}

This work was supported by National Natural Science Foundation of China (Grant No. 81572055) and the funder finished the study design.

\section{Availability of data and materials}

The datasets generated and analysed during the current study are included in this published article and its supplementary information files. More datasets are not publicly available due to the need for further reaserch, but are available from the corresponding author on reasonable request.

\section{Ethics approval and consent to participate}

The study protocol was approved by the ethics committee of The First Affiliated Hospital of Chongqing Medical University. Written consents informed were obtained from all participants.

\section{Consent for publication}

Not applicable.

\section{Competing interests}

The authors declare that they have no competing interests.

\section{Author details}

${ }^{1}$ Department of Laboratory Medicine, The First Affiliated Hospital of Chongqing Medical University, No.1 Youyi Road, Yuzhong District, Chongqing 400016, People's Republic of China. ${ }^{2}$ Department of Laboratory Medicine, Dianjiang People's Hospital of Chongqing, No.116 North Street, Guixi Street, Dianjiang County, Chongqing 408300, People's Republic of China.

Received: 20 October 2019 Accepted: 6 April 2020

Published online: 15 April 2020

\section{References}

1. Cai J, Zhao C, Du Y, Zhang Y, Zhao M, Zhao Q, et al. Comparative efficacy and tolerabilityof probiotics for antibiotic-associated diarrhea: systematic review with network meta-analysis. United European Gastroenterol J. 2018; 6(2):169-80.

2. Schäffler $H$, Breitrück $A$. Clostridium difficile-from colonization to infection. Front Microbiol. 2018;9:646.

3. Lessa FC, Mu Y, Bamberg WM, Beldavs ZG, Dumyati GK, Dunn JR, et al. Burden of Clostridium difficile infection in the United States. N Engl J Med. 2015;372(9):825-34.

4. Wiegand PN, Nathwani D, Wilcox MH, Stephens J, Shelbaya A, Haider S. Clinical and economic burden of Clostridium difficile infection in Europe: a systematic review of healthcare- facility-acquired infection. J Hosp Infect. 2012;81(1):1-14.

5. Borren NZ, Ghadermarzi S, Hutfless S, Ananthakrishnan AN. The emergence of Clostridium difficile infection in Asia: a systematic review and metaanalysis of incidence and impact. PLoS One. 2017;12(5):e0176797.

6. Tang C, Cui L, Xu Y, Xie L, Sun P, Liu C, et al. The incidence and drug resistance of Clostridium difficile infection in Mainland China: a systematic review and meta-analysis. Sci Rep. 2016:6, 37865.

7. Liu XS, Li WG, Zhang WZ, Wu Y, Lu JX. Molecular characterization of Clostridium difficile isolates in China from 2010 to 2015. Front Microbiol. 2018;9:845.

8. Qin J, Dai Y, Ma X, Wang Y, Gao Q, Lu H, et al. Nosocomial transmission of Clostridium difficile genotype ST81 in a general teaching Hospital in China traced by whole genome sequencing. Sci Rep. 2017;7(1):9627.

9. Jia H, Du P, Yang H, Zhang Y, Wang J, Zhang W, et al. Nosocomial transmission of Clostridium difficile ribotype 027 in a Chinese hospital, 2012-2014, traced by whole genome sequencing. BMC Genomics. 2016;17: 405.

10. Leffler DA, Lamont JT. Clostridium diffcile infection. N Engl J Med. 2015;372: 1539-48.

11. Griffiths D, Fawley W, Kachrimanidou M, Bowden R, Crook DW, Fung R, et al. Multilocus sequence typing of Clostridium difficile. J Clin Microbiol. 2010; 48(3):770-8.

12. Indra A, Huhulescu $S$, Schneeweis $M$, Hasenberger $P$, Kernbichler $S$, FiedlerA WG, Allerberger F, Kuijper EJ. Characterization of Clostridium difficile isolates using capillary gel electrophoresis-based PCR ribotyping. J Med Microbiol. 2008;57(Pt 11):1377-82.

13. Clinical and Laboratory Standards Institute. Methods for antimicrobial susceptibility testing of anaerobic bacteria. Approved standard M11-A8. Wayne: CLSI; 2016.

14. CLSI. Performance standards for antimicrobial susceptibility testing; M10029th edition. Wayne: Clinical and Laboratory Standards Institute; 2019.

15. Lidan C, Linhai L, Yang L, Zhaohui S, Xiaoyan H, Yuling S. Molecular characterization and antimicrobial susceptibility of tcdA-negative Clostridium difficile isolates from Guangzhou, China. Diagn Microbiol Infect Dis. 2016;84(4):361-5.

16. The European Committee on Antimicrobial Susceptibility Testing. Breakpoint tables for interpretation of MICs and zone diameters. Version 10. 0; 2010. http://www.eucast.org.

17. Fawley WN, Knetsch CW, MacCannell DR, Harmanus C, Du T, Mulvey MR, et al. Development and validation of an internationally-standardized, highresolution capillary gel-based electrophoresis PCR-ribotype protocol for Clostridium difficile. PLoS One. 2015;10(2):e0118150.

18. Sun JD, Huang SF, Yang SS, Pu SL, Zhang CM, Zhang LP. Impact of carbapenem heteroresistance among clinical isolates of invasive Escherichia coli in Chongqing, southwestern China. Clin Microbiol Infect. 2015;21(5):469. e1-10. 
19. Zou XX, Fang Z, Min R, Bai X, Zhang Y, Xu D, et al. Is nationwide special campaign on antibiotic stewardship program effective on ameliorating irrational antibiotic use in China? Studyon the antibiotic use of specialized hospitals in China in 2011-2012. J Huazhong Univ Sci Technolog Med Sci. 2014;34(3):456-63.

20. Zhou FF, Wu S, Klena JD, Huang HH. Clinical characteristics of Clostridium difficile infection in hospitalized patients with antibiotic-associated diarrhea in a university hospital in China. Eur J Clin Microbiol Infect Dis. 2014;33(10): 1773-9.

21. Gillespie D, Hood K, Bayer A, Carter B, Duncan D, Espinasse A, et al. Antibiotic prescribing and associated diarrhoea: a prospective cohort study of care home residents. Age Ageing. 2015;44(5):853-60

22. Wong S, Santullo P, Hirani SP, Kumar N, Chowdhury JR, García-Forcada A, et al. Use of antibiotics and the prevalence of antibiotic-associated diarrhea inpatients with spinal cord injuries: an international, multicentre study. J Hosp Infect. 2017;97(2):146-52.

23. Hawkey PM, Marriott C, Liu WE, Jian ZJ, Gao Q, Ling TK, et al. Molecular epidemiology ofClostridium difficile infection in a major Chinese hospital: an underecognized problem in Asia? J Clin Microbiol. 2013;51(10):3308-13.

24. Nasiri MJ, Goudarzi M, Hajikhani B, Ghazi M, Goudarzi H, Pouriran R. Clostridioides (Clostridium) difficile infection in hospitalized patients with antibiotic associated diarrhea: a systematic review and meta-analysis. Anaerobe. 2018;50:32-7.

25. Wang LF, Cao J, Li CY, Zhang LP. IL-27/IL-27 Receptor Signaling Provides Protection in Clostridium difficile-Induced Colitis. J Infect Dis. 217(2):198-207.

26. Xu Q, Chen Y, Gu S, Lv T, Zheng B, Shen P, et al. Hospital-acquired Clostridium difficile infection in mainland China: a seven-year (2009-2016) retrospective study in a large university hospital. Sci Rep. 2017;7(1):9645.

27. Kamboj M, Brite J, Aslam A, Kennington J, Babady NE, Calfee D, et al. Artificial differences in Clostridium difficile infection rates associated with disparity in testing. Emerg Infect Dis. 2018;24(3):584-7.

28. Wiep Klaas Smits, Dena Lyras, D. Borden Lacy, Mark H. Wilcox, Ed J. Kuijper. Clostridium difficile infection. Nat Rev Dis Primers. 2,16020; doi: https://doi. org/10.1038/nrdp.2016.20.

29. Khanafer N, Vanhems P, Barbut F, Luxemburger C, CDI01 study group, et al. Factors associated with Clostridium difficile infection: A nested case-control study in a three year prospective cohort. Anaerobe. 2017;44:117-23.

30. Huang $\mathrm{H}$, Wu S, Chen $\mathrm{R}$, Xu S, Fang $\mathrm{H}$, Weintraub $\mathrm{A}$, et al. Risk factors of Clostridium difficile infections among patients in a university hospital in Shanghai, China. Anaerobe. 2014;30:65-9.

31. Lawes T, Lopez-Lozano J-M, Nebot CA, Macartney G, Subbarao-Sharma R, Wares KD, Sinclair C, Gould IM. Effect of a national 4C antibiotic stewardship intervention on the clinical and molecular epidemiology of Clostridium difficile infections in a region of Scotland: a non-linear time-series analysis. Lancet Infect Dis. 2017:17(2):194-206.

32. Slimings C, Riley TV. Antibiotics and hospital-acquired Clostridium difficile infection: update of systematic review and meta-analysis. J Antimicrob Chemother. 2014;69:881-91.

33. Jin D, Luo Y, Huang C, Cai J, Ye J, Zheng Y, et al. Molecular epidemiology of Clostridium difficile infection in hospitalized patients in eastern China. J Clin Microbiol. 2017;55(3):801-10.

34. Wang XM, Zhou XD, Hesketh T. Massive misuse of antibiotics by university students in China: a cross-sectional survey. Lancet. 2016;388(Suppl1):S94.

35. Fischer $\mathrm{N}$, Relman DA. Clostridium difficile, aging, and the gut: can microbiome rejuvenation keep us young and healthy? J Infect Dis. 2018; 217(2):174-6.

36. Li H, Li WG, Zhang WZ, Yu SB, Liu ZJ, Zhang X, Wu Y, Lu JX. Antibiotic resistance of clinicalisolates of Clostridioides difficile in China and its association with geographical regions and patient age. Anaerobe. 2019;6: 102094.

37. Cheng JW, Xiao M, Kudinha T, Kong F, Xu ZP, Sun LY, Zhang L, Fan X, Xie $X L, X u$ YC. Molecular Epidemiology and Antimicrobial Susceptibility of Clostridium difficile Isolates from a University Teaching Hospital in China. Front Microbiol. 2016;7:1621.

38. Wang R, Suo L, Chen HX, Song LJ, Shen YY, Luo YP. Molecular epidemiology and antimicrobial susceptibility of Clostridium difficile isolated from the Chinese People's liberation Army general Hospital in China. Int J Infect Dis. 2018;67:86-91

39. Liao F, Li W, Gu W, Zhang W, Liu X, Fu X, Xu W, Wu Y, Lu J. A retrospective study of community-acquired Clostridium difficile infection in Southwest China. Sci Rep. 2018;8(1):3992.
40. Chen YB, Gu SL, Shen P, Lv T, Fang YH, Tang LL, Li L. Molecular epidemiology and antimicrobial susceptibility of Clostridium difficile isolated from hospitals during a 4-year period in China. J Med Microbiol. 2018;67(1): 52-9.

41. Chen R, Feng Y, Wang X, Yang J, Zhang X, Lü X, et al. Whole genome sequences of three clade 3 Clostridium difficile strains carrying binary toxingenes in China. Sci Rep. 2017;7:43555.

42. Luo Y, Zhang W, Cheng JW, Xiao M, Sun GR, Guo CJ, et al. Molecular epidemiology of Clostridium difficile in two tertiary care hospitals in Shandong Province, China. Infect Drug Resist. 2018;11:489-500.

43. Krutova M, Kinross P, Barbut F, Hajdu A, Wilcox MH, Kuijper EJ. Survey contributors. How to: surveillance of Clostridium difficile infections. Clin Microbiol Infect. 2018;24(5):469-75. https://doi.org/10.1016/j.cmi.2017.12.008 Epub 2017 Dec 20.

\section{Publisher's Note}

Springer Nature remains neutral with regard to jurisdictional claims in published maps and institutional affiliations.

\section{Ready to submit your research? Choose BMC and benefit from:}

- fast, convenient online submission

- thorough peer review by experienced researchers in your field

- rapid publication on acceptance

- support for research data, including large and complex data types

- gold Open Access which fosters wider collaboration and increased citations

- maximum visibility for your research: over $100 \mathrm{M}$ website views per year

At BMC, research is always in progress.

Learn more biomedcentral.com/submissions 